\title{
Nonunitary mixing: current constraints and new ambiguity
}

\author{
Omar Miranda ${ }^{* \dagger}$ \\ Departamento de Física, \\ Centro de Investigación y de Estudios Avanzados del IPN, \\ Apdo. Postal 14-740 07000 Mexico, DF, Mexico \\ E-mail: omrefis.cinvestav.mx
}

\begin{abstract}
Neutrino physics has been under continuos progress along several decades from now. As a consequence, the main parameters for neutrino mixing are under constant improvement and entering into a precision era that will allow testing for the unitarity of the mixing matrix in the leptonic sector. Here we discuss the triangular parametrization as a useful tool in this context, and we give a brief discussion on the current status on the restriction to its parameters.
\end{abstract}

Neutrino Oscillation Workshop

4 - 11 September, 2016

Otranto (Lecce, Italy)

\footnotetext{
* Speaker.

${ }^{\dagger}$ Work based on [1]
} 
Particle physics is living an interesting period where all the building blocks of the Standard Model have been found by the experiment. Besides the need for precision measurements, there are strong motivations to search for new physics due to, for example, the unification theories and the dark matter and dark energy problems. In the neutrino sector, the needs for an explanation of the tiny non-zero neutrino mass has been the motivation for several popular theories beyond the standard model, being the seesaw mechanism the most popular of them. It is predicted in these models that new neutrino states would exist. There are many phenomenological consequences of these hypotheses and here we focus particularly on the non-unitarity of the standard $3 \times 3$ neutrino mass, due to the existence of the extra neutrino states. In particular, we describe a new parametrization that has been recently proposed [1] and has been found useful in dealing with neutrino oscillations $[2,4,3]$. We will also discuss briefly some of the current constraints as well as the role of the new phases that appears if we depart from the standard $3 \times$ unitary case.

We start by considering the most general description for $n$ neutrino states. In this case, we will need a unitary matrix $U^{n \times n}$ describing the neutrino diagonalization matrix. It is known that this matrix can be fully described using the symmetric parametrization[5], given by the products of complex matrices $\omega_{i j}$ that describe, effectively, $2 \times 2$ rotations in a given $i j$-axis:

$$
U^{n \times n}=\omega_{n-1 n} \omega_{n-2 n} \ldots \omega_{1 n} \omega_{n-2 n-1} \omega_{n-3 n-1} \ldots \omega_{1 n-1} \ldots \omega_{23} \omega_{13} \omega_{12} .
$$

When considering the particular case of three light neutrinos plus $n-3$ extra heavy neutrino states, it is useful to look at this matrix as [7]

$$
U^{n \times n}=\left(\begin{array}{cc}
N & S \\
V & T
\end{array}\right),
$$

with $N$ a $3 \times 3$ matrix describing the light observed neutrino sector. As discussed in [1], this matrix $N$ can be parametrized as

$$
N=N^{N P} U=\left(\begin{array}{ccc}
\alpha_{11} & 0 & 0 \\
\alpha_{21} & \alpha_{22} & 0 \\
\alpha_{31} & \alpha_{32} & \alpha_{33}
\end{array}\right) U .
$$

Here, $U$ is the usual $3 \times 3$, unitary, leptonic mixing matrix. This parametrization gives a general description of a non-unitary $3 \times 3$ mixing matrix. Besides, it has also the appealing characteristic that we can have the $\alpha_{i j}$ expressed in terms of the mixing angles and phases of the complete matrix [1]. The departure from unitarity in this parametrization will be contained in these $\alpha_{i j}$ parameters; three of them will be real and will be equal to one in the unitary limit (the diagonal ones). The other three parameters will be complex and will tend to zero.

As a consequence of the possible existence of heavier states, the weak decays will not longer obey universality since the heavier mass eigenstates will not participate in these processes. The effect would translate into different values of the effective Fermi constant for the given decays. For instance, in the muon decay, the Fermi constant would have the form

$$
G_{\mu}=G_{F} \sqrt{\left(N N^{\dagger}\right)_{11}\left(N N^{\dagger}\right)_{22}}=G_{F} \sqrt{\alpha_{11}^{2}\left(\alpha_{22}^{2}+\left|\alpha_{21}\right|^{2}\right)} .
$$

Using current experimental values, constraints on the universality parameters can be obtained, for example from the CKM matrix elements [8] as well as from pion decay [9]. In particular, we have 
reported the following constraints from univerality, at 90\% C.L.,

$$
\begin{aligned}
1-\alpha_{11}^{2} & <0.0130 \\
1-\alpha_{22}^{2}-\left|\alpha_{21}\right|^{2} & <0.0012 .
\end{aligned}
$$

Stronger constraints can be obtained using more recent values. Moreover, from the constraints on the diagonal parameters, it is possible to obtain restrictions on the non-diagonal ones. However, it is also important to obtain independent direct constraints on these non-diagonal parameters. In this direction, the only direct constraint for $\left|\alpha_{21}\right|$ may com from the NOMAD experiment [10], that gives the restriction

$$
\alpha_{11}^{2}\left|\alpha_{21}\right|^{2} \leq 0.0007 \text { (90\% C.L.) }
$$

In the case of neutrino oscillation experiments, the triangular parametrization gives a simple description of the non-unitary case. A simple example is the conversion probability from muon to electron neutrino that, in the vacuum case, will be given by the approximate expression [1]

$$
P_{\mu e}=\alpha_{11}^{2}\left|\alpha_{21}\right|^{2}+\left(\alpha_{11} \alpha_{22}\right)^{2} P_{\mu e}^{3 \times 3}+\alpha_{11}^{2} \alpha_{22}\left|\alpha_{21}\right| P_{\mu e}^{I}
$$

where, $P_{\mu e}^{3 \times 3}$ is the well-known three-neutrino conversion probability [11]; the constant term $\alpha_{22}^{2}\left|\alpha_{21}\right|^{2}$ represents the zero-distance effect that comes from the non-orthogonality of the neutrino weak eigenstates [12], and the term $P_{\mu e}^{I}$ introduce a new dependence on neutrino energy and on an additional CP phase coming from the new sector. This term will depend on two different phases: the standard CP phase $\delta$ characterizing three-neutrino oscillations, and an additional CP phase associated to the new physics, $\phi$, given by the argument of the complex parameter

$$
\begin{aligned}
P_{\mu e}^{I} & =-2\left[\sin \left(2 \theta_{13}\right) \sin \theta_{23} \sin \left(\frac{\Delta m_{31}^{2} L}{4 E_{v}}\right) \sin \left(\frac{\Delta m_{31}^{2} L}{4 E_{v}}+\delta_{C P}+\phi\right)\right] \\
& -\cos \theta_{13} \cos \theta_{23} \sin \left(2 \theta_{12}\right) \sin \left(\frac{\Delta m_{21}^{2} L}{2 E_{v}}\right) \sin (\phi) .
\end{aligned}
$$

The presence of this additional phase may lead to a degeneracy in the conversion probability, especially for big values of $\left|\alpha_{21}\right|$ [13]. The reason for this is simple; for the values of $L / E$ used by long baseline neutrino experiments, there will bee an interplay between the standard $\delta_{C P}$ phase and the new phase. These two phases will be related mainly by the expression:

$$
\begin{aligned}
2 \alpha_{11}^{2} \alpha_{22}^{2} & \sin \theta_{13} \sin \theta_{23} \sin \Delta_{31} \sin 2 \Delta_{21} \times \\
& {\left[\sin 2 \theta_{12} \cos \theta_{23} \cos \left(\Delta_{31}+\delta_{C P}\right)\right.} \\
- & \left.2 \frac{\cos \theta_{13}}{\alpha_{22}} \frac{\left|\alpha_{21}\right|}{\sin 2 \Delta_{21}} \sin \left(\Delta_{31}+\delta_{C P}+\phi\right)\right] .
\end{aligned}
$$

It will be of interest for long baseline neutrino experiments to obtain direct model-independent constraints on the non-diagonal elements of the triangular matrix, both on their magnitudes as well as in their phases $[4,14,15]$. At the same time, it will also be important to verify that the standard CP violating phase will be well determined despite the possible ambiguity with new $\mathrm{CP}$ phases [15]. 


\section{Acknowledgements}

I would like to thank my collaborators from Ref. [1] were these proceedings are based. This work is supported by the CONACyT grant 166639 and Red-FAE.

\section{References}

[1] F. J. Escrihuela, D. V. Forero, O. G. Miranda, M. Tortola and J. W. F. Valle, Phys. Rev. D 92, no. 5, 053009 (2015) Erratum: [Phys. Rev. D 93, no. 11, 119905 (2016)] doi:10.1103/PhysRevD.93.119905, 10.1103/PhysRevD.92.053009 [arXiv:1503.08879 [hep-ph]].

[2] D. Dutta, P. Ghoshal and S. K. Sehrawat, arXiv:1610.07203 [hep-ph].

[3] M. Blennow, P. Coloma, E. Fernandez-Martinez, J. Hernandez-Garcia and J. Lopez-Pavon, arXiv:1609.08637 [hep-ph].

[4] C. S. Fong, H. Minakata and H. Nunokawa, arXiv:1609.08623 [hep-ph].

[5] J. Schechter and J. W. F. Valle, Phys. Rev. D 22, 2227 (1980). doi:10.1103/PhysRevD.22.2227

[6] W. Rodejohann and J. W. F. Valle, Phys. Rev. D 84, 073011 (2011) doi:10.1103/PhysRevD.84.073011 [arXiv:1108.3484 [hep-ph]].

[7] H. Hettmansperger, M. Lindner and W. Rodejohann, JHEP 1104, 123 (2011) doi:10.1007/JHEP04(2011)123 [arXiv:1102.3432 [hep-ph]].

[8] A. Abada, A. M. Teixeira, A. Vicente and C. Weiland, JHEP 1402, 091 (2014) doi:10.1007/JHEP02(2014)091 [arXiv:1311.2830 [hep-ph]].

[9] A. Abada, D. Das, A. M. Teixeira, A. Vicente and C. Weiland, JHEP 1302, 048 (2013) doi:10.1007/JHEP02(2013)048 [arXiv:1211.3052 [hep-ph]].

[10] P. Astier et al. [NOMAD Collaboration], Phys. Lett. B 570, 19 (2003) doi:10.1016/j.physletb.2003.07.029 [hep-ex/0306037].

[11] H. Nunokawa, S. J. Parke and J. W. F. Valle, Prog. Part. Nucl. Phys. 60, 338 (2008) doi:10.1016/j.ppnp.2007.10.001 [arXiv:0710.0554 [hep-ph]].

[12] J. W. F. Valle, Phys. Lett. B 199, 432 (1987). doi:10.1016/0370-2693(87)90947-6

[13] O. G. Miranda, M. Tortola and J. W. F. Valle, Phys. Rev. Lett. 117, no. 6, 061804 (2016) doi:10.1103/PhysRevLett.117.061804 [arXiv:1604.05690 [hep-ph]].

[14] D. Dutta and P. Ghoshal, JHEP 1609, 110 (2016) doi:10.1007/JHEP09(2016)110 [arXiv:1607.02500 [hep-ph]].

[15] Escrihuela et al. in preparation. 